Claremont Colleges

Scholarship@ Claremont

All HMC Faculty Publications and Research

HMC Faculty Scholarship

9-1-2008

\title{
Synthesis and Assembly of Nonspherical Hollow Silica Colloids Under Confinement
}

Stephanie H. Lee

Cornell University

Sharon J. Gerbode

Harvey Mudd College

Bettina S. John

Cornell University

Angie K. Wolfgang

Cornell University

Fernando A. Escobedo

Cornell University

See next page for additional authors

\section{Recommended Citation}

S. Lee, S. J. Gerbode, B. John, A. Wolfgang, I. Cohen, F. Escobedo, and C. Liddell, "Synthesis and assembly of nonspherical hollow silica colloids under confinement” J. Mat. Chem., 18(41), 4912- 4916 (2008). doi: 10.1039/B812406J

This Article is brought to you for free and open access by the HMC Faculty Scholarship at Scholarship @ Claremont. It has been accepted for inclusion in All HMC Faculty Publications and Research by an authorized administrator of Scholarship @ Claremont. For more information, please contact scholarship@cuc.claremont.edu. 
Authors

Stephanie H. Lee, Sharon J. Gerbode, Bettina S. John, Angie K. Wolfgang, Fernando A. Escobedo, Itai Cohen, and Chekesha M. Liddell 


\section{Journal of}

Materials Chemistry

www.rsc.org/materials

Volume 18 | Number 41 | 7 November 2008 | Pages 4881-5028

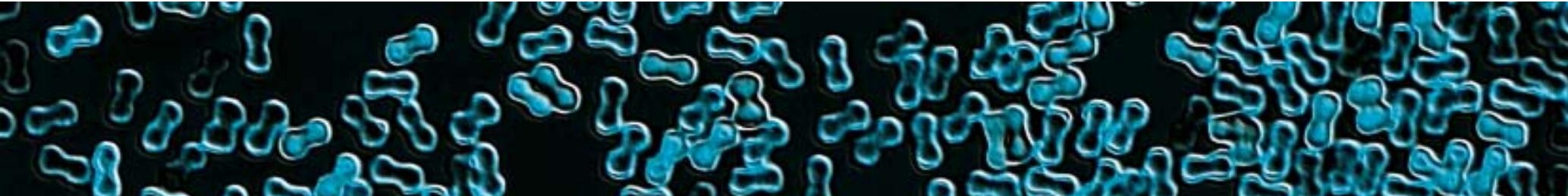

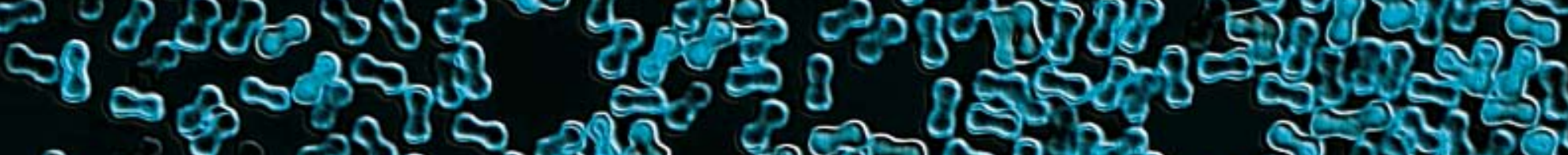

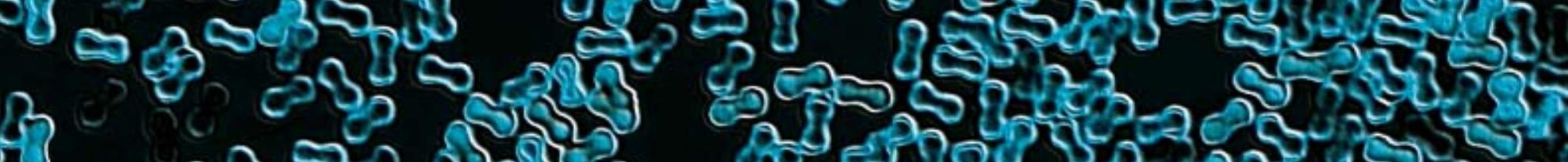

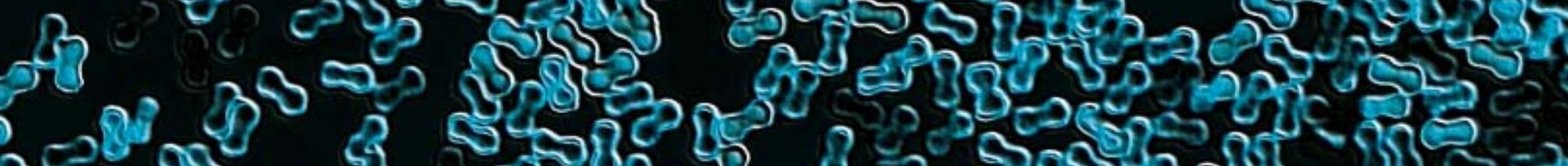

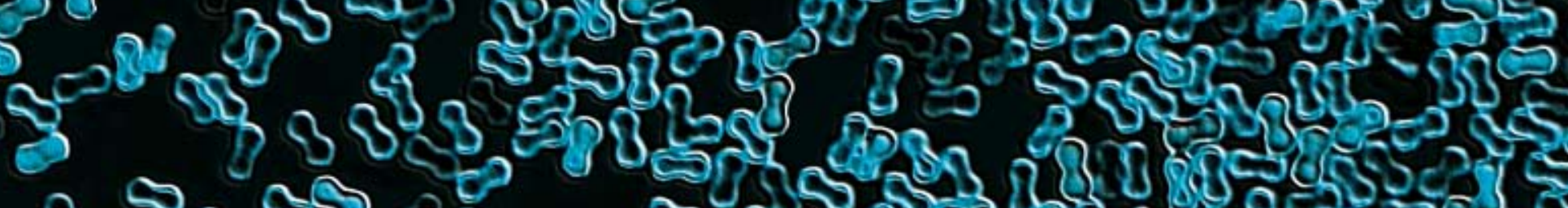

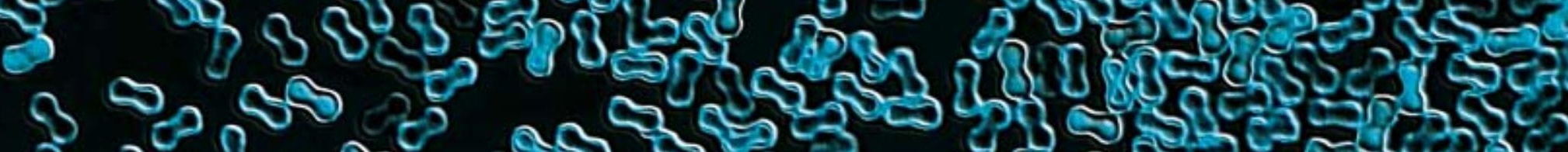

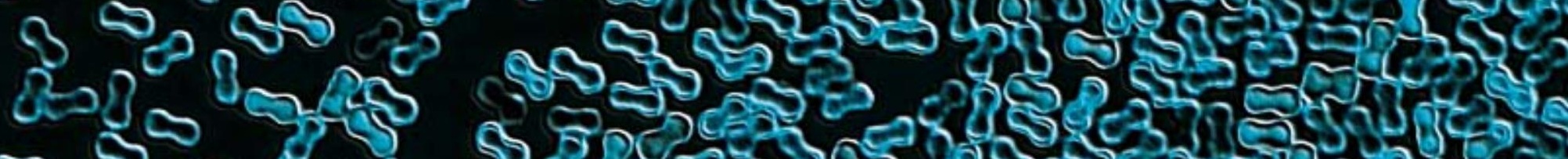

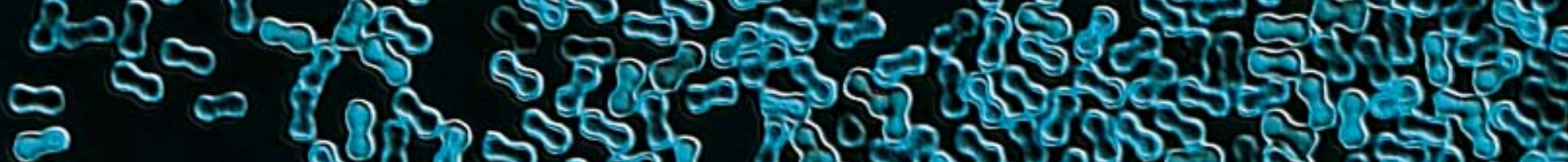

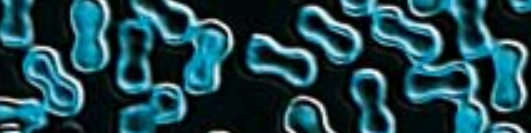

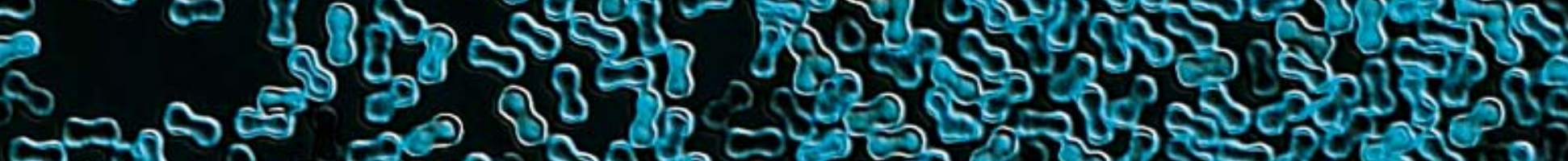

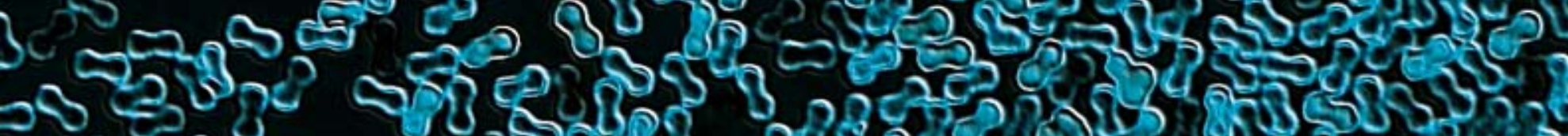

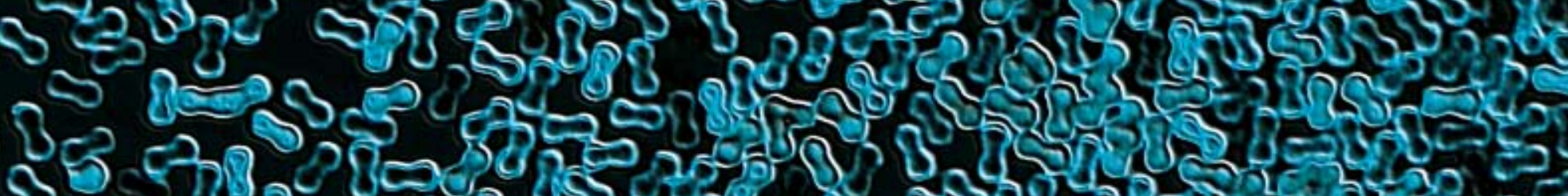

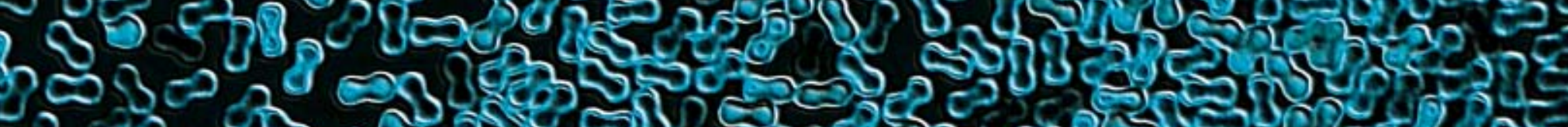
8 0 -

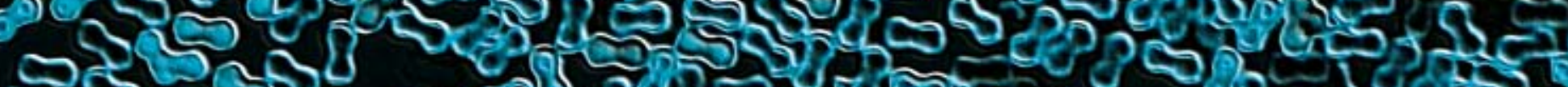

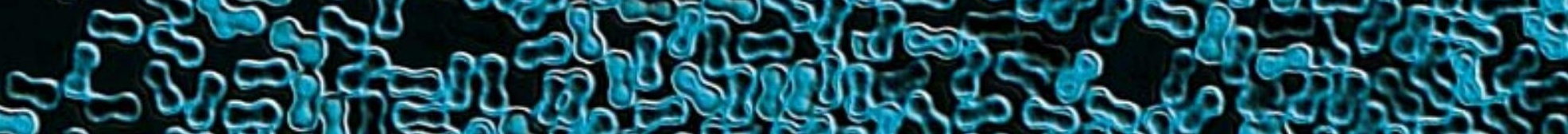

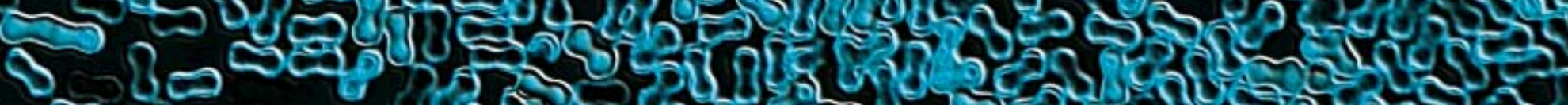
a

\section{PAPER}

Sang Cheon Lee et al.

Designing a highly bioactive 3D

bone-regenerative scaffold by surface

immobilization of nano-hydroxyapatite 


\title{
Synthesis and assembly of nonspherical hollow silica colloids under confinement
}

\author{
Stephanie H. Lee, ${ }^{* a}$ Sharon J. Gerbode, ${ }^{b}$ Bettina S. John, ${ }^{c}$ Angie K. Wolfgang, ${ }^{b}$ Fernando A. Escobedo, ${ }^{c}$ \\ Itai Cohen ${ }^{b}$ and Chekesha M. Liddell ${ }^{* a}$
}

\author{
Received 18th July 2008, Accepted 18th August 2008 \\ First published as an Advance Article on the web 22nd September 2008 \\ DOI: $10.1039 / b 812406 j$
}

\begin{abstract}
Hard peanut-shaped colloids were synthesized and organized into a degenerate crystal (DC), a phase previously observed only in simulations. In this structure, particle lobes tile a triangular lattice while their orientations uniformly populate the three underlying crystalline directions.
\end{abstract}

Colloidal self-assembly has long been cited as a means to create periodic materials for photonic, optoelectronic, solar cell, and sensing applications. The technique is attractive because it holds promise for the simple microfabrication of low-cost, large-scale devices. Nevertheless, structures that are accessible with spherical particles have generally been restricted to the rather common hexagonal closepacked or face-centered cubic forms, except in the case of bidisperse systems (i.e., size and charge). ${ }^{1,2}$ The lack of diversity is a result of isotropic interactions in spherical particles, which fail to capture the selective functionalities and geometries required to encode the ordering of more elaborate assemblies. In contrast are sophisticated structures such as icosahedral virus capsids and herringbone pentacene thin films, which use protein sub-units or small molecules as their structural motifs. These architectures inspire a strategy to increase complexity in traditional colloids by introducing symmetry-breaking features in the particle shape or interparticle potential. Essential to this idea is the ability to impart specific assembly instructions to the colloidal building blocks so that materials with enhanced functional properties can be made.

Approaches to induce directional interactions include using shapeselective depletion forces, which in disk-shaped colloids led to the formation of columnar structures, ${ }^{3}$ or dipolar forces, which in spherical particles led to the formation of body-centered tetragonal assemblies. ${ }^{4}$ Intricate building blocks consisting of spherical particles with directional patches have also been synthesized recently. ${ }^{5}$ Simulations predict that under the right conditions these particles can form a diamond structure, ${ }^{6}$ one of the best candidates for a complete photonic bandgap material. Other theoretical predictions have indicated that a rich number of phases can be realized through the selfassembly of nonspherical particles. For instance, heteronuclear dimers were predicted to condense into a methyl chloride-like structure, given a sufficient electric dipole moment per particle. ${ }^{7}$ Hard spherocylinders of different aspect ratios also ordered into stable

${ }^{a}$ Department of Materials Science and Engineering, Cornell University, Ithaca,NY, 14853, USA.E-mail: hsl24@cornell.edu; cliddell@ccmr. cornell.edu

${ }^{b}$ Physics Department, Cornell University, Ithaca, NY, 14853, USA

${ }^{c}$ Department of Chemical and Biomolecular Engineering, Cornell University, Ithaca, NY, 14853, USA states that included orientationally ordered solids with AAA or ABC stacking and a plastic crystal phase. ${ }^{8}$ These simulations illustrate the potential to produce colloidal assembly analogues by design, especially when viewed in light of recent progress in colloidal synthesis techniques, which yield particles of exotic shape, functionality, and high monodispersity. ${ }^{9-14}$ In spite of these advances, relatively few welldefined structures have been made thus far from complex-shaped colloids. ${ }^{15,16}$

The present work highlights the synthesis of peanut-shaped particles with hard dimer-like interactions and demonstrates their assembly into ordered structures under confinement. The particles were prepared through a multi-step templating process that produced hollow, fluorescent silica shells amenable to direct visualization with a confocal microscope. To synthesize the core hematite particles $\left(\alpha-\mathrm{Fe}_{2} \mathrm{O}_{3}\right)$, a condensed ferric hydroxide gel was first aged under hydrothermal conditions ${ }^{17}$ (Fig. 1a). A rhodamine-functionalized silica shell was then grown onto the hematite surface using modified

(a)

(c)


(b)
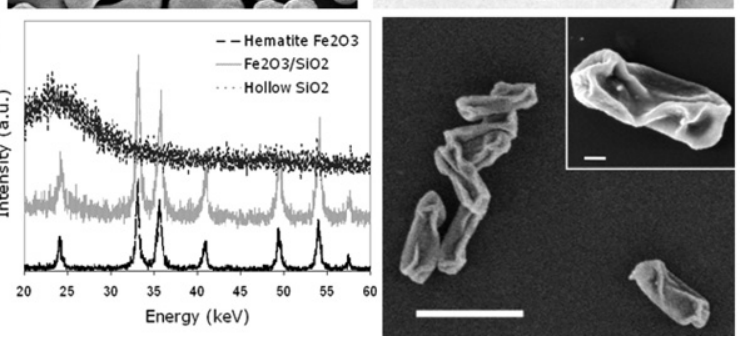

(d)

Fig. 1 Fluorescent silica particles. a) FE-SEM image of monodisperse hematite colloids from which hollow particles were templated. Scale bar, 2.5 microns. b) TEM bright-field micrograph of intact silica shells (thickness, $65 \mathrm{~nm}$ ). Scale bar, 1.5 microns. c) XRD pattern of hematite particles (bottom, black), hematite-silica core-shell particles (middle, light gray), and hollow silica shells (top, dark gray). The bottom two show peaks corresponding to crystalline hematite (JCPDS card no. 33-0664) while the top exhibits only an amorphous signature indicative of complete etching. d) Collapsed particles (thickness, $28 \mathrm{~nm}$ ) below the critical thickness, $t_{\mathrm{c}}$. Scale bar, 2 microns. The inset displays a highmagnification view of the collapsed morphology. Scale bar, $400 \mathrm{~nm}$. 
sol-gel chemistry. ${ }^{18}$ This protocol produced $95 \%$ pure dimer particles with final dimensions of $2.82 \mu \mathrm{m} \times 1.36 \mu \mathrm{m}$, and a coefficient of variation of $4.7 \%$. The thickness of the shells ranged between 20 and $150 \mathrm{~nm}$ and was tuned via suitable selection of the silica precursor concentration and feed times. Since hematite has a high index of refraction that hinders confocal imaging due to scattering, and a weak magnetization that alters the assembly process, the core was sacrificed through selective etching using concentrated hydrochloric acid (Fig. 1b). Complete removal of the hematite was confirmed by XRD (Fig. 1c).

Peanut-shaped shells were found to be structurally stable only when the thinnest coating regions passed a critical shell thickness of $46 \pm 10 \mathrm{~nm}$. Below this thickness, hollow dimers crumpled without fracture and lost their intended shape (Fig. 1d). A high-magnification FE-SEM micrograph is shown in the inset (Fig. 1d) to illustrate the details of the collapsed morphology. Increasing the silica precursor concentration led to thicker shells, though coatings were limited to $\sim 65 \mathrm{~nm}$ depositions in a single step to prevent the undesirable nucleation of smaller, secondary silica particles. The particles were sterically stabilized and suspended in an aqueous solution which yielded nearly hard body interactions. If the cores were left intact, however, dipolar attractions due to the antiferromagnetic hematite led to chains and nematic-like structures. This phenomenon will be discussed elsewhere.

Here, the ordering of a dense monolayer of dimers was investigated for hollow particles confined in a wedge geometry cell (Fig. 2a). Particle area fraction was controlled by gently tilting the cell so that the dimers slowly sedimented into the region of interest. Before imaging, the confinement cell was laid flat to allow for equilibration over the entire field of view. The spontaneous organization of the colloids at high area fraction is shown in the confocal micrograph of Fig. 2b. Although the positions of the particle centers are aperiodic, an image captured at a focal plane that grazes only the particle tips reveals triangular ordering in the lobes (Fig. 2c). This symmetry is quantified by the form of the lobe pair correlation function, $g(r)$, taken over an area of $4800 \mu \mathrm{m}^{2}$ (Fig. 3a). As demonstrated by the trimodal distribution in Fig. 3b, peanut-shaped colloids oriented with equal probability along the three crystalline axes defined by the lobes. In addition, because the interparticle angles occur predominantly at intervals of $\pi / 3$, they can be well characterized by the orientation correlation function
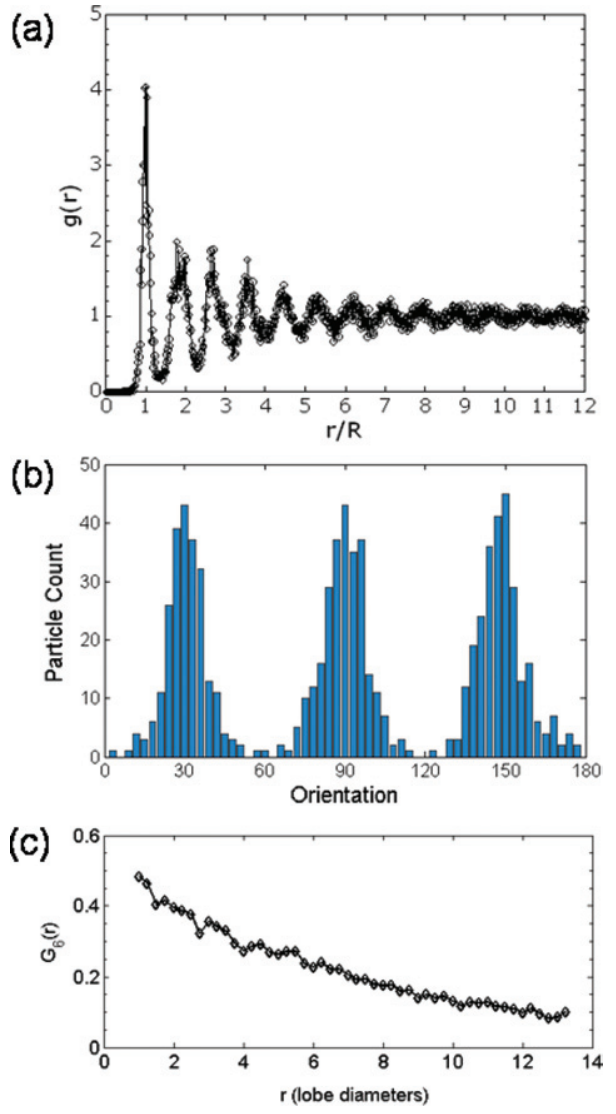

Fig. 3 Structural characterization. a) Lobe radial distribution function, $g(r)$. b) Histogram of particle orientations taken from various crystallites. The first peak measured from each grain was shifted to $\theta=30^{\circ}$ to illustrate the trimodal distribution clearly. c) The orientation correlation function, $G_{6}(r)$, for the experimental system. The curve shows a non-zero but decreasing long-range value, indicating that although interparticle angles are ordered close to multiples of $\pi / 3$, there may be crystalline defects that cause the observed decay.

$$
G_{6}(r)=\left\langle\sum_{i \leq i<j \leq N} \cos \left(6 \Delta \theta_{i j}\right) \cdot \delta\left(r_{i j}-r\right)\right\rangle
$$

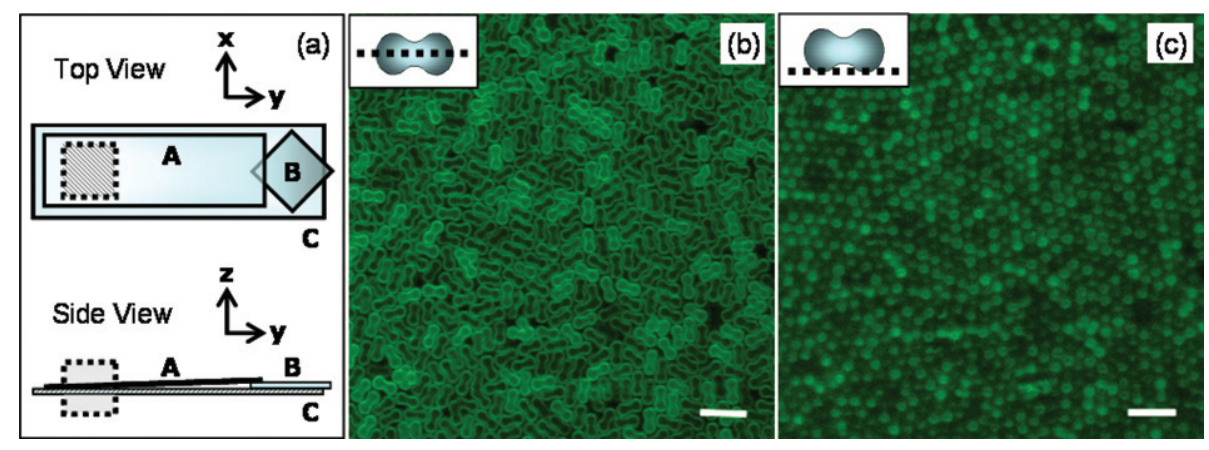

Fig. 2 Confocal microscopy of dense monolayers of peanut-shaped particles. a) Schematic of wedge-shaped confinement cell used to constrain the nonspherical dimer colloids. Part A represents a long coverslip, B a spacer coverslip, and C the microscope slide substrate. The hatched area indicates a region where the $z$-height is roughly $1.5 \mu \mathrm{m}$. b) Confocal image of a dense monolayer of colloids taken across the equatorial plane (inset, schematic view). Scale bar, 5 microns. c) Triangular ordering of the lobes is revealed when an image is captured at a focal plane that just grazes the particle lobes (inset, schematic). Scale bar, 5 microns. 
(a)



(b)

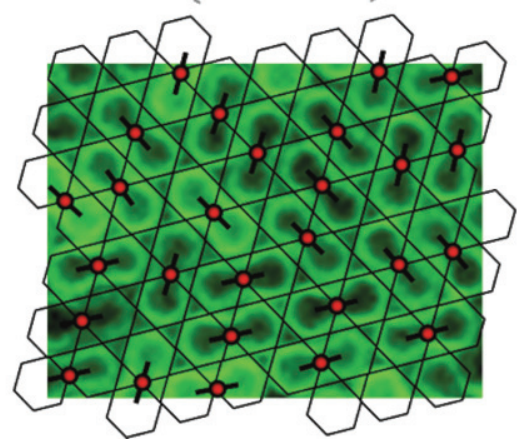

Fig. 4 Kagomé construction of the degenerate crystal (DC). a) A schematic showing the structure of the DCs observed in prior simulations. ${ }^{19}$ Red dots represent the particle centers of mass, white circles show the Kagomé lattice sites, and dashed grey lines indicate the three crystalline axes (after ref. 20). b) Kagomé construction applied to the experimental data with peanut-shaped colloids. The overlaid Kagomé net is a guide for the eye. where $\Delta \theta_{i j}$ is the difference in orientation between the $i$ th and $j$ th particle, and $r_{i j}$ is the distance between their centers of mass. The function $G_{6}(r)$ gives spatial information on the dimer orientations and expresses their degree of ordering to $\Delta \theta_{i j}$ values of $n \pi / 3$, where $n$ is an integer. Given perfect lobe positional order and dimer orientations, $G_{6}(r)$ should equal unity. In the experimental system (Fig. 3c), $G_{6}(r)$ exhibits lower values due to the angular spread in $\Delta \theta_{i j}$ (Fig. 3b). It also displays a decaying long-range orientational order in the dimer axes, which may be suggestive of crystalline defects or grain boundaries.

The observed structure is reminiscent of the degenerate crystal (DC), ${ }^{19}$ which has thus far been seen only in MC simulations of dimers with aspect ratio $\leq 2$. As with our colloidal particles, in the simulated DCs, dimer lobes tile a triangular lattice while particle orientations populate one of three crystalline directions. Such a phase exhibits strong configurational degeneracy, and was determined numerically to be the thermodynamically-stable phase. ${ }^{20}$ An additional feature characterizing DCs is that the dimer centers irregularly decorate a Kagomé lattice so that exactly one site is occupied in each unit hexagon (Fig. 4a). Although the particles in the current study are elongated with aspect ratio 2.074, rather than shortened as in the MC simulations, the particle centers were found to satisfy this Kagomé requirement (Fig. 4b).

A distinct difference between the simulated single-grain DCs and the observed structures was that the latter always contained many crystallites with an average dimension of $10 \pm 1$ lobe diameters. This small grain size is reflected in the lobe pair correlation function, whose peaks decay beyond this length scale (Fig. 3a). Fig. 5a highlights the defect distribution in a typical sample using a Voronoi (a)

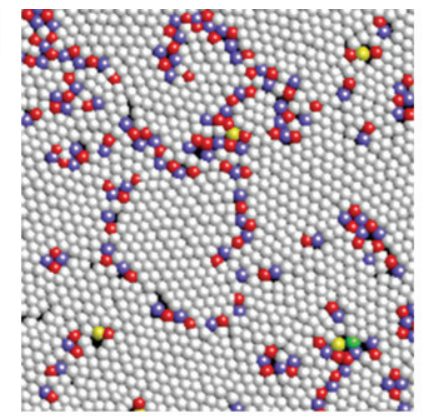

(c)

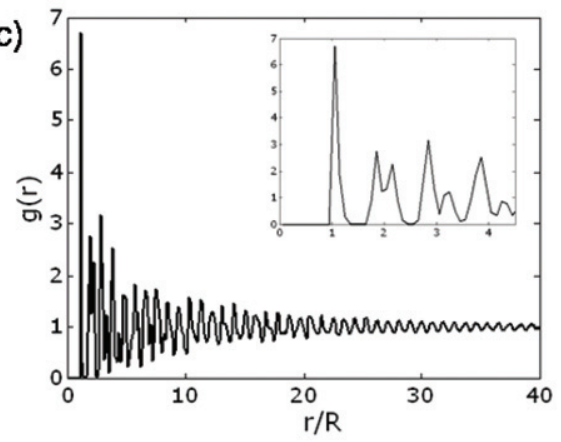

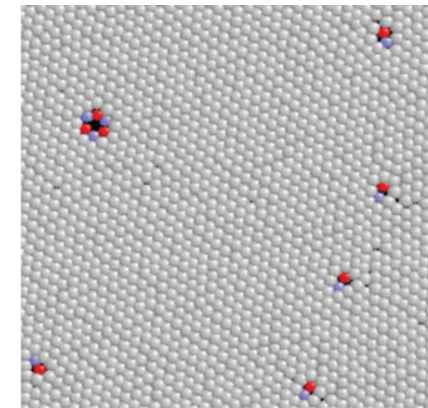

(b)

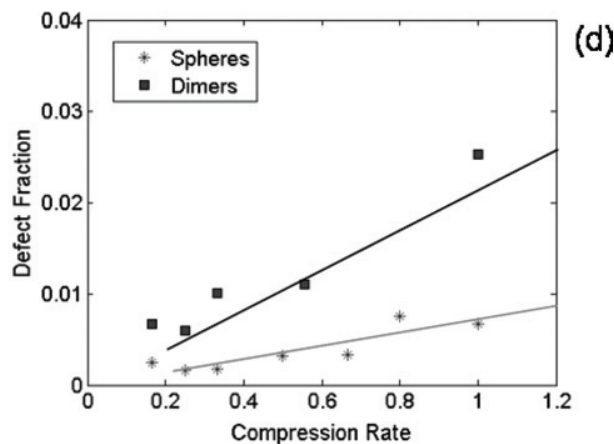

Fig. 5 Defects and Monte Carlo simulations. a) Voronoi reconstruction for the experimental data, which shows $N=2394$ particle lobes color-coded by their coordination number. Defects, consisting of lobes with a coordination number other than six, are highlighted with non-white colors. b) Voronoi reconstruction from MC simulations at a compression rate of 0.17 . A magnified region of the sample area $(N=7200$ lobes $)$ is shown for clarity. c) Lobe pair correlation function associated with the simulation set from $b$. Inset, magnified view of the first few peaks. d) Comparison of the defect fraction in crystals made of peanut-shaped dimers (black squares) versus spherical particles (grey asterisks) at various compression rates. Trend lines were added to guide the eye. 
reconstruction, in which every particle lobe is colored according to its coordination number. This schematic reveals that while some defects are isolated, many coalesce into string-like grain boundaries separating DC crystallites of different orientations. The mean fraction of defects is $15 \%$, and of those, $61 \%$ are located on the grain boundaries. While both types of defects were mobile, their total numbers remained nearly constant over a time scale of weeks. The observation of defect mobility in the experimental DC is in stark contrast to defects in crystalline monolayers of hard spheres with comparable particle size and area fractions, which anneal over a time scale of hours.

The importance of defect mobility lies in its crucial role in determining many material properties ranging from yield and plastic flow to work hardening and fatigue. In crystals of spheres the motion of defects is achieved through simple particle rearrangements. In the dimer system, however, each lobe is permanently attached to one of its neighbors, and thus a rearrangement necessitates not only a translational but also an orientational change. To illustrate the significance of this lobe pairing on defect annealing, and consequently on defect fractions, Monte Carlo (MC) simulations of crystalline monolayers of spheres and dimers formed under compression were compared. Ideal monodisperse particles were modeled in an isothermal ensemble starting from a disordered state at low density. The system was then compressed by incrementally increasing the pressure until it crystallized (Fig. 5b,c). In these simulations, compression rates were varied by changing the number of $\mathrm{MC}$ cycles used for equilibration at each pressure. Fig. 5d illustrates that crystals of dimers had a higher fraction of defects than crystals of spheres at all compression rates. Furthermore, this effect is more pronounced at higher compression rates, where the fraction of defects increases for dimers but remains nearly zero for spheres. These MC simulations, in conjunction with the experiments, suggest that defect dynamics in DCs may be considerably more constrained than those in crystals of spheres. Future investigations should help to clarify the differences in defect transport that lead to the observed disparity in annealing times. In addition, it would be of interest to probe whether DCs have long-range orientational order, but short-range translational order, as is characteristic of a hexatic phase. Such ordering is challenging to identify, however, particularly when dealing with polycrystalline systems.

The present report illustrates that proper selection and synthetic control of colloidal building blocks can open opportunities for the self-assembly of materials with novel structures, as exemplified by the observed DCs. Further studies, such as transition path sampling simulations and nucleation and growth experiments, should help to elucidate the mechanisms of the isotropic to DC phase transition. It may also be possible to design new assemblies of these particles by introducing further interactions with templated substrates. Observations of colloidal assembly on such surfaces may provide intuition for atomic scale processes, such as the physisorption and phase transitions of diatomic molecules on graphite/Au/Ag films. ${ }^{21}$ It has been shown that pairing constraints in DCs lead to interesting mechanical properties such as a low Poisson ratio. ${ }^{22}$ If $3 \mathrm{D}$ versions of these phases exhibit a similarly low Poisson ratio, they could be valuable as materials for the manufacture of piezoelectric composites, used commonly in low-frequency actuators of sonar arrays and seismic monitors. ${ }^{23}$ Most importantly, our studies of these simple anisotropic particles hint at the diversity of assemblies that can be constructed using nonspherical colloids. As such, it represents a positive step towards achieving molecular mimicry, whereby self-organization of systems with novel properties is driven by codes embedded within the constituent building blocks.

\section{Experimental}

\section{Particle synthesis}

Core peanut-shaped $\alpha-\mathrm{Fe}_{2} \mathrm{O}_{3}$ particles were prepared by a gel-sol method as described previously. ${ }^{17}$ In a typical synthesis, $50 \mathrm{~mL}$ of $\mathrm{FeCl}_{3}(2.0 \mathrm{M})$ was mixed with $45 \mathrm{~mL}$ of $\mathrm{NaOH}(6.0 \mathrm{M})$ and $5 \mathrm{~mL}$ of $\mathrm{Na}_{2} \mathrm{SO}_{4}(0.6 \mathrm{M})$ and aged in a preheated oven $\left(100^{\circ} \mathrm{C}\right)$ for 8 days. $\mathrm{A}$ layer of rhodamine isothiocyanate (RITC)-modified silica was then coated onto the $\alpha-\mathrm{Fe}_{2} \mathrm{O}_{3}$ cores via a modified Stöber synthesis ${ }^{18}$ conducted under sonochemical conditions (probe power $14 \mathrm{~W}, 30$ ${ }^{\circ} \mathrm{C}$ ). To make shells approximately $65 \mathrm{~nm}$ thick, the reaction mixture comprised: $0.4 \%(\mathrm{w} / \mathrm{v})$ hematite powder, $0.25 \%(\mathrm{v} / \mathrm{v})$ rhodamine dye solution, 7.1 M deionized water, 0.92 M ammonia, and $17.4 \mathrm{mM}$ tetraethylorthosilicate (TEOS) added batch-wise to isopropyl alcohol medium. Hollow silica shells were made by selectively removing the core with concentrated hydrochloric acid $(18 \%)$ after surface modification with poly(vinylpyrrolidone) (PVP, $40000 \mathrm{MW}$ ). Before use, colloids were titrated to neutral $\mathrm{pH}$ and washed via repeated centrifugation and decanting before being re-dispersed into deionized water.

\section{Sample preparation}

The wedge-shaped confinement cell was assembled by fixing three sides of a $22 \times 50 \mathrm{~mm}$ glass coverslip (VWR, No. 1/1/2) to a large microscope slide and propping the last side up with a $22 \times 22 \mathrm{~mm}$ spacer coverslip. Three sides of the coverslip were sealed with Norland UV optical glue, cured overnight, and reinforced with fiveminute epoxy. To load the particles, a dilute peanut-shaped colloidal dispersion was injected into the cell at the open end via a micropipette tip and was sealed with epoxy. The wedge cell was titled $10-60^{\circ}$ from horizontal to control the area fraction of particles within the region of interest by allowing the colloids to sediment. After sufficient densification, the cell was laid flat to re-establish equilibrium. Particles were visualized directly using a confocal microscope.

\section{System characterization}

SEM images were collected using an LEO 1550 field emission scanning electron microscope (FE-SEM) at 0.5-3 kV. TEM micrographs were captured with an FEI Tecnai T12 Spirit Twin transmission electron microscope at an operating voltage of $120 \mathrm{kV}$. To determine the $\mathrm{SiO}_{2}$ shell thickness, values were measured from 50 points on hollow shells using TEM images, with the uncertainty given by the standard deviation. XRD spectra were obtained using $\mathrm{Cu} \mathrm{K} \alpha$ radiation on a Scintag $\theta-\theta$ diffractometer equipped with an intrinsic germanium detector. For confocal data, a Zeiss LSM 5 LIVE inverted laser scanning confocal microscope with an excitation wavelength of $532 \mathrm{~nm}$ was used.

\section{Monte Carlo (MC) simulations}

In the Monte Carlo (MC) simulations, monolayers of peanut-shaped or spherical particles were compressed until they crystallized. The peanut-shaped dimers $(N=3600)$ were modeled as two spheres whose centers were located a distance of 1.075 particle diameters 
apart. The samples were kept at isothermal conditions throughout the simulations. Compression was achieved by increasing the pressure in steps and allowing for equilibration at each step using translation and rotation of randomly chosen dimers, with an acceptance rate based on the Metropolis criteria. The compression rate was initially defined as the ratio of the Gibbs free energy difference, $\Delta G$, between initial and final pressures $P_{1}$ and $P_{2}$, to the total number of MC cycles in millions of cycles. The value of $\Delta G$ was chosen such that it was always the same and thus provided the same thermodynamic driving force toward compression for the dimer and spherical particles, even though crystallization for the two systems occur at different area fractions. Because $\Delta G$ is constant, we then simply redefined the compression rate as the reciprocal of the millions of MC cycles used to go from $P_{1}$ and $P_{2} . \Delta \mathrm{G}$ was found from $\Delta G=\int_{P_{1}}^{P_{2}} A \mathrm{~d} P$, where $A$ is the total surface area of the system, and $P_{1}$ and $P_{2}$ were chosen such that: (i) $P_{1}\left(P_{2}\right)$ was well inside the isotropic (crystal) branch of the equation of state, and (ii) $\Delta G$ between $P_{1}$ and the isotropic-crystal transition pressure $P^{*}$, and $\Delta G$ between $P^{*}$ and $P_{2}$ were the same across both the dimer and sphere systems. The integrations were evaluated based on the equilibrium equation of state data. For the spheres: $P_{1}=5$ (surface coverage, $\mathrm{SC}=0.601$ ) and $P_{2}=14.8$ (equilibrium $\mathrm{SC} \sim 0.785)$; For the dimers: $P_{1}=3.4(\mathrm{SC} \sim 0.624)$ and $P_{2}=13.0$ (equilibrium $\mathrm{SC}=0.843$ ). The compression rate was maintained at a low enough value to allow the systems to approach the final equilibrium surface coverage and crystallize. Since crystals of spheres always formed single grains, simulation results were also restricted to those that formed single DC grains in order to compare the two systems on an equal basis.

\section{Acknowledgements}

We gratefully acknowledge funding of this work by the National Science Foundation (Career Award No. DMR-0547976, DMR0606040, DMR-0553719) and the American Chemical Society PRF Grant \#45525-AC7. Additionally, this work was performed in part at facilities of the Cornell Center for Materials Research (NSF MRSEC, DMR-0520404).

\section{Notes and references}

1 E. V. Shevchenko, D. V. Talapin, N. A. Kotov, S. O'Brien and C. B. Murray, Structural diversity in binary nanoparticle superlattices, Nature, 2006, 439, 55-59.

2 M. E. Leunissen, C. G. Christova, A.-P. Hynninen, C. P. Royall, A. I. Campbell, A. Imhof, M. Dijkstra, R. van Roij and A. van Blaaderen, Ionic colloidal crystals of oppositely charged particles, Nature, 2005, 437, 235-240.

3 S. Badaire, C. Cottin-Bizonne, J. W. Woody, A. Yang and A. D. Stroock, Shape selectivity in the assembly of lithographically designed colloidal particles, J. Am. Chem. Soc., 2007, 129, 40-41.
4 A. Yethiraj and A. van Blaaderen, A colloidal model system with an interaction tunable from hard sphere to soft and dipolar, Nature, 2003, 421, 513-517.

5 Y. S. Cho, G.-R. Yi, S.-H. Kim, S.-J. Jeon, M. T. Elsesser, H. K. Yu, S.-M. Yang and D. J. Pine, Particles with coordinated patches or windows from oil-in-water emulsions, Chem. Mater., 2007, 19, 3183-3193.

6 Z. L. Zhang, A. S. Keys, T. Chen and S. C. Glotzer, Self-assembly of patchy particles into diamond structures through molecular mimicry, Langmuir, 2005, 21, 11547-11551.

7 S. C. Gay, P. D. Beale and J. C. Rainwater, Solid-liquid equilibrium of dipolar heteronuclear hard dumbbells in a generalized van der Waals theory: Application to methyl chloride, J. Chem. Phys., 1998, 109, 6820-6827.

8 P. Bolhuis and D. Frenkel, Tracing the phase boundaries of hard spherocylinders, J. Chem. Phys., 1997, 106, 666-687.

9 J. W. Kim, R. J. Larsen and D. A. Weitz, Synthesis of nonspherical colloidal particles with anisotropic properties, J. Am. Chem. Soc., 2006, 128, 14374-14377.

10 D. Dendukuri, D. C. Pregibon, J. Collins, T. A. Hatton and P. S. Doyle, Continuous-flow lithography for high-throughput microparticle synthesis, Nat. Mater., 2006, 5, 365-369.

11 Y. S. Cho, G.-R. Yi, J.-M. Lim, S.-H. Kim, V. N. Manoharan, D. J. Pine and S.-M. Yang, Self-organization of bidisperse colloids in water droplets, J. Am. Chem. Soc., 2005, 127, 15968-15975.

12 P. M. Johnson, C. M. van Kats and A. van Blaaderen, Synthesis of colloidal silica dumbbells, Langmuir, 2005, 21, 11510-11517.

13 V. N. Manoharan, M. T. Elsesser and D. J. Pine, Dense packing and symmetry in small clusters of microspheres, Science, 2003, 301, 483487.

14 C. M. Liddell and C. J. Summers, Monodispersed ZnS dimers, trimers, and tetramers for lower symmetry photonic crystal lattices, Adv. Mater., 2003, 15, 1715-1719.

15 E. B. Mock and C. F. Zukoski, Determination of Static Microstructure of Dilute and Concentrated Suspensions of Anisotropic Particles by Ultra-Small-Angle X-ray Scattering, Langmuir, 2007, 23, 8760-8771.

16 I. D. Hosein and C. M. Liddell, Convectively assembled asymmetric dimer-based colloidal crystals, Langmuir, 2007, 23, 10479-10485.

17 T. Sugimoto, M. M. Khan and A. Muramatsu, Preparation of monodisperse peanut-type alpha- $\mathrm{Fe}_{2} \mathrm{O}_{3}$ particles from condensed ferric hydroxide gel, Colloids Surf., A, 1993, 70, 167-169.

18 A. van Blaaderen and A. Vrij, Synthesis and characterization of colloidal dispersions of fluorescent, monodisperse silica spheres, Langmuir, 1992, 8, 2921-2931.

19 K. W. Wojciechowski, D. Frenkel and A. C. Branka, Nonperiodic solid phase in a two-dimensional hard-dimer system, Phys. Rev. Lett., 1991, 66, 3168-3171.

$20 \mathrm{~K}$. W. Wojciechowski, Monte Carlo simulations of highly anisotropic two-dimensional hard dumbbell-shaped molecules: Nonperiodic phase between fluid and dense solid, Phys. Rev. B, 1992, 46, 26-39.

21 M. F. Toney, R. D. Diehl and S. C. Fain, Rotational epitaxy of a nontriangular structure: The delta-phase of oxygen physisorbed on graphite, Phys. Rev. B, 1983, 27, 6413-6417.

$22 \mathrm{~K}$. V. Tretiakov and K. W. Wojciechowski, Elastic properties of the degenerate crystalline phase of two-dimensional hard dimers, J. Non-Cryst. Solids, 2006, 352, 4221-4228.

23 A. Perry, C. R. Bowen and S. W. Mahon, Finite Element Modelling of 3-3 Piezocomposites, Scr. Mater., 1999, 41, 1001-1007. 